hep-ph/9812395

LA-UR-98-5729

\title{
Generalized Schwinger Mass Formula
}

\author{
L. Burakovsky, ${ }^{*}$ P.R. Page ${ }^{\dagger}$ and T. Goldman ${ }^{\ddagger}$ \\ Theoretical Division, MS B283 \\ Los Alamos National Laboratory \\ Los Alamos, NM 87545, USA
}

\begin{abstract}
We generalize Schwinger's original mass formula to the case of an additional isosinglet mixing with the nonet mesons. We then make further generalization to either (i) an arbitrary number of additional isosinglets mixing with nonet mesons, or (ii) an arbitrary number of mesons mixing with an additional isosinglet. In the former case, we present an explicit relation, while in the latter case, we show by numerical example that the new mass formula is only weakly affected by the inclusion of additional mesons, and hence holds with good accuracy for each of the $3 \times 3$ mass sub-matrices.
\end{abstract}

Key words: Schwinger's formula, mass matrix, isosinglet-mesons mixing PACS: 12.39.Mk, 12.40.Yx, 12.90.+b, 14.40.-n

\footnotetext{
*E-mail: BURAKOV@T5.LANL.GOV

${ }^{\dagger}$ E-mail: PRP@LANL.GOV

${ }^{\ddagger}$ E-mail: GOLDMAN@T5.LANL.GOV
} 


\section{Introduction}

Schwinger's original mass formula [1] has attained celebrity due to its generality in a universe with only nine mesons, under the assumption that up, down and strange quarks may be treated the same.

If there were only up and down quarks which interact identically via the strong force, the $u \bar{u}$ and $d \bar{d}$ states of the hadron spectrum could be resolved into degenerate isovector and isosinglet states A. Although this requires more than isospin invariance, we know it is consistent (for mesons) with the details of QCD dynamics as there are no spin-isospin correlations here to produce color magnetic spin interaction induced splittings, at least for the lowest Fock components.

The isovector masses can be regarded as physical since they undergo no further mixing. Therefore, in the Schwinger formula, the primitive (bare) isosinglet masses can be equated with the (physical) isovector masses. Isosinglet states are invariant under rotation of up into down quarks and have the flavour structure $n \bar{n} \equiv(u \bar{u}+d \bar{d}) / \sqrt{2}$. Enter the strange quark: Primitive $s \bar{s}$ states, the masses of which are not a priori known, can mix with the primitive $n \bar{n}$ states to create physical states. Schwinger's formula pertains to the case where there is one $n \bar{n}$ and one $s \bar{s}$ primitive state, and where the strange quark behaves the same as the up and down quarks when the two primitive states couple to each other ( $\mathrm{SU}(3)$ symmetry).

The formula has the benefit, except for the primitive $s \bar{s}$ mass, of referring only to experimentally measurable meson masses, and not intractable couplings.

In this Letter we extend Schwinger's formula to include additional isosinglets and relax the $\mathrm{SU}(3)$ assumption of Schwinger. This enables a more model independent analysis of the presence of isosinglets. In the case where the isosinglet is built mainly from gluons (a glueball), this extension is of central significance as some experimentally discovered isosinglet zero angular momentum states [2] are very widely accepted to exhibit mixing with a glueball [3, 4].

Schwinger's formula

$$
[3 \tilde{M}(s \bar{s})-2 M(s \bar{s})-M(n \bar{n})][3 \tilde{M}(n \bar{n})-2 M(s \bar{s})-M(n \bar{n})]+2[M(s \bar{s})-M(n \bar{n})]^{2}=0
$$

relates the masses of the physical $(\tilde{M}(s \bar{s}), \tilde{M}(n \bar{n}))$ and primitive $(M(s \bar{s}), M(n \bar{n}))$ mesons. In this formula, and in other relations below, $M$ 's stand either for the mass or mass squared.

The formula may be derived in the following way [5]: For a meson nonet, the isosinglet $q \bar{q}$ mass matrix modified by the inclusion of the mixing amplitudes $A$, which are here assumed flavor-symmetric, for simplicity,

$$
\mathcal{M}^{(2 \times 2)}=\left(\begin{array}{cc}
M(s \bar{s})+A & \sqrt{2} A \\
\sqrt{2} A & M(n \bar{n})+2 A
\end{array}\right),
$$

\footnotetext{
${ }^{1}$ In the pseudoscalar sector the axial anomaly and instantons can lift the degeneracy [5]. In the scalar sector only instantons lift the degeneracy, and in other sectors these effects are not significant [5].
} 


\begin{tabular}{|c|c|c|c|c|}
\hline$J^{P C}$ & States & I & II & III \\
\hline $1^{--}$ & $\rho, K^{*}, \omega, \phi$ & $1013.0 \pm 0.5$ & $1014.5 \pm 0.4$ & $1017.8 \pm 5.8$ \\
\hline $2^{++}$ & $a_{2}(1320), K_{2}^{*}(1430), f_{2}(1270), f_{2}^{\prime}(1525)$ & $1541.7 \pm 5.3$ & $1539.4 \pm 5.5$ & $1539.9 \pm 10.6$ \\
\hline $2^{-+}$ & $\pi_{2}(1670), K_{2}(1770), \eta_{2}(1645), \eta_{2}(1870)$ & $1870.7 \pm 26.0$ & $1869.1 \pm 25.3$ & $1869 \pm 24$ \\
\hline $3^{--}$ & $\rho_{3}(1690), K_{3}^{*}(1780), \omega_{3}(1670), \phi_{3}(1850)$ & $1863.1 \pm 9.2$ & $1862.3 \pm 9.0$ & $1863.2 \pm 16.1$ \\
\hline
\end{tabular}

Table I. Comparison of the values for $M(s \bar{s})$ given by Eq. (1) for linear and squared masses (in columns I and II, respectively), and the quark model motivated relation discussed in the text for linear masses (in column III). All the mass values are given in $\mathrm{MeV}$. All the mass values are taken from ref. [6]. Uncertainties represent the combination of experimental uncertainties and the scale of electromagnetic splittings.

is diagonalized by the masses of the physical mesons: $\mathcal{M}^{(2 \times 2)} \Longrightarrow \operatorname{diag}(\tilde{M}(s \bar{s}), \tilde{M}(n \bar{n}))$. Writing down the trace and determinant conditions for the matrix (2) and eliminating $A$ from them leads to the formula (1).

In Table I we compare the predictions of Schwinger's formula for the mass of the primitive $s \bar{s}$ state, based on the masses of the primitive $n \bar{n}$ state assumed to be the mass of the isovector, and the two physical states, with the predictions of the quark model motivated linear mass relation $M(n \bar{n})+M(s \bar{s})=2 M(s \bar{n})$, where we use the mass of the isodoublet for $M(s \bar{n})$.

Schwinger's formula reflects the limitations on changes of masses from primitives to physical states that can occur only by mixing. To the extent that one may identify masses of primitives, by extracting mixing angles or using model assumptions (as above), the formula agrees well with data in most cases.

We interject a comment on the primitives. They are not simply the result of suppressing quark loop contributions. For example, in quenched lattice QCD, OZI rule violating decays of $s \bar{s}$ states into two light quark-antiquark pairs may still occur. Perhaps a useful analogy may be found in the issue of CP-violating kaon decays there are both mass mixing induced and intrinsic CP-violating decays. Thus here it is insufficient simply to extract the mixing angle to the primitives by determining from the experimental decay rates which combinations of the physical states would not violate the OZI rule. One must allow for the analog of the intrinsic rule violating decays.

Because of this, it continues to be much easier to define primitives on the basis of some model assumption, such as that the light isoscalar primitive is equal in mass to the light isovector state. Further, the notion that the light-strange mixed quark primitive averages the masses of the pure light and pure strange primitives receives adequate support from a wide range of model calculations dating back to the earliest days of the quark model through to the present refined potential and string models. The success of the Schwinger formula with these definitions of the primitives establishes the existence of primitives with these properties. At this stage the glueball is "integrated out" in the formula.

The agreement of Schwinger's formula with data is, however, problematical, as the mixing must pass through glueballs determined by QCD dynamics. Here we shall examine such mass relations by explicitly including the nearest glueball intermediate 
states to describe the mixing and complete the phenomenological picture, noting that when the glueballs are near to the mesons one should not be able to "integrate out" their effects. We show that if the single intermediate glueball state is only modestly more massive than mesons in the light sector, then the $2 \times 2$ and $3 \times 3$ matrices give almost identical results. This is to be expected in the large glueball mass limit, but we find it develops surprisingly early - just above $2 \mathrm{GeV}$. We describe how the situation generalizes in the presence of multiple meson nonets.

The validity of Schwinger's original formula (1) should be expected to break down in cases when additional isosinglets mix strongly with the primitive mesons. Such additional isosinglets may be glueballs or hidden flavor heavy quark mesons with the quantum numbers of the nonet mesons. In such cases, one has to consider a larger mass matrix, $N \times N, N>2$, for which an analog of the original formula (1) may be expected to exist.

In this paper we derive such an analog of Schwinger's original mass formula (1) in the case of any number of additional isosinglets (we need not specify their nature for our present purposes).

\section{Generalized Schwinger mass formula}

Let us first note that a field theory in general, and QCD in particular, does not have a mass matrix corresponding to composite states. Indeed, the diagonalization of a mass matrix corresponds to a linear transformation of fields that diagonalizes the Lagrangian, which can only be done for Lagrangians which are quadratic in fields, e.g., the Klein-Gordon Lagrangian, with quadratic interactions. A mass matrix appears therefore only in effective field theories of this type. However, precisely this type of an effective meson theory may be expected to exist for QCD. In fact, the emergence of such a theory was shown in [7] through the bosonization of QCD. Hence, we assume the existence of an effective theory that describes the mixing of nonet mesons with additional isosinglets.

We start with the case of one additional isosinglet. Assume, for simplicity, that an additional isosinglet (glueball or hidden flavor heavy quark meson), $i$, couples to a pair of primitive (nonet) mesons. Then, we have the following $3 \times 3$ hermitian mass matrix where, in addition to the quark mixing amplitudes, we have the amplitudes of the $i-q \bar{q}$ mixing (the couplings) which we denote by $B$ :

$$
\mathcal{M}^{(3 \times 3)}=\left(\begin{array}{ccc}
M(i) & B & B r \\
B^{*} & M(s \bar{s})+A & A r \\
B^{*} r^{*} & A r^{*} & M(n \bar{n})+A|r|^{2}
\end{array}\right),
$$

where $M(i)$ is the mass of the primitive $i$. From now on, in contrast to Eq. (2), we allow for possible violation of exact flavor $\mathrm{SU}(3)$ symmetry in terms of arbitrary $r$. The $\mathrm{SU}(3)$ limit will then correspond to $r=\sqrt{2}$. The form of this mass matrix is motivated in ref. [8]. The mass matrix (3) was also discussed in [9], and its $\mathrm{SU}(3)$ symmetric version in [10]. 
Note that in Eq. (3), as well as in Eq. (2), $A$ must be real. With $B$ and $r$ both real the matrix (3) is the most general parametrization of $3 \times 3$ (real) symmetric matrix, since it contains 6 independent parameters.

The mass matrix (3) is diagonalized by the masses of the three physical states, $\mathcal{M}^{(3 \times 3)} \Longrightarrow \operatorname{diag}(\tilde{M}(i), \tilde{M}(s \bar{s}), \tilde{M}(n \bar{n}))$, which are determined from the three eigenvalue equations (which follow from $\operatorname{Det}\left(\mathcal{M}^{(3 \times 3)}-\lambda I\right)=0$ ). Eliminating $A$ and $B$ from the eigenvalue equations leads, upon some algebra, to the formula

$$
\begin{gathered}
{\left[\left(1+|r|^{2}\right) \tilde{M}(i)-|r|^{2} M(s \bar{s})-M(n \bar{n})\right]\left[\left(1+|r|^{2}\right) \tilde{M}(s \bar{s})-|r|^{2} M(s \bar{s})-M(n \bar{n})\right]} \\
\times\left[\left(1+|r|^{2}\right) \tilde{M}(n \bar{n})-|r|^{2} M(s \bar{s})-M(n \bar{n})\right] \\
+\left[\left(1+|r|^{2}\right) M(i)-|r|^{2} M(s \bar{s})-M(n \bar{n})\right]|r|^{2}[M(s \bar{s})-M(n \bar{n})]^{2}=0,
\end{gathered}
$$

which is the generalized Schwinger mass formula for the case of a meson nonet with an additional isosinglet. We note that the new formula does not depend on either the values of $A$ or $B$, just as the original formula does not depend on the value of $A$.

Under the conditions that either

(i) $\left(1+|r|^{2}\right) M(i) \gg|r|^{2} M(s \bar{s})+M(n \bar{n})$ (see Eq. (4)), or

(ii) $|B| \ll A$,

so that $\tilde{M}(i) \approx M(i)$, the generalized Schwinger formula (4) (with $r=\sqrt{2}$ ) reduces to the original one, Eq. (1).

In the case (iii) $A=0$,

i.e., no direct coupling between mesons, one can combine the new formula (4) with one of the eigenvalue equations (the trace condition for the matrix (3))

$$
\tilde{M}(i)+\tilde{M}(s \bar{s})+\tilde{M}(n \bar{n})=M(i)+M(s \bar{s})+M(n \bar{n})
$$

in order to determine two unknown masses. Under the assumption that (iii) is the realistic case for meson spectroscopy, i.e., that the observed meson mass spectra result from coupling of the primitive $q \bar{q}$ states through an additional isosinglet, one can calculate the masses of two physical states, e.g., $\tilde{M}(i), \tilde{M}(s \bar{s})$, as functions of $M(i)$, using the known $M(n \bar{n}), M(s \bar{s})$. In Figs. 1,2 we compare $\tilde{M}(s \bar{s})$ calculated in this way with the range allowed for $\tilde{M}(s \bar{s})$ by experimental data [6], for the $J^{P C}=3^{--}$ multiplet (here we use Eqs. (4),(5) for linear masses). One sees that the value calculated lies within the range allowed by data for a rather large bare mass of an additional isosinglet, $\gtrsim 2 \mathrm{GeV}$.

Note that all isosinglet masses above 2.5 and $2 \mathrm{GeV}$, respectively, are consistent with $\tilde{M}(s \bar{s})$ from experiment, so that the mass of an additional isosinglet cannot be uniquely determined from current experimental data. Thus, the procedure described above (only) fixes the lower bound on the mass of an additional state $(i)$.

\footnotetext{
${ }^{2}$ The other meson nonets can be analyzed in a similar way. The case of the $J^{P C}=0^{++}$has been studied in detail in ref. [12.
} 


\section{$3 \quad$ Further generalization of the new Schwinger mass formula}

The most general case for a mass formula of the type (4) is evidently the case of the mixing of arbitrary numbers of both mesons and additional isosinglets with common $J^{P C}$. This most general case can be decomposed into the following two cases: (i) an arbitrary number of additional isosinglets (glueballs and hidden flavor heavy quark mesons) mixing with one pair of (nonet) mesons built from $u, d, s$ quarks, and (ii) an arbitrary number of pairs of excited mesons (with common $J^{P C}$ ) built from $u, d, s$ quarks mixing with one isosinglet (glueball or hidden flavor heavy quark meson). We now examine these two cases in more detail.

\subsection{The case of an arbitrary number of additional isosinglets}

In this case the new Schwinger mass formula has an explicit further generalization, as follows.

Consider the mixing of $(N-2)$ extra isosinglets with a pair of nonet mesons and introduce, as previously, the corresponding bare masses $M\left(i_{1}\right), \ldots, M\left(i_{N-2}\right)$, couplings $B_{1}, \ldots, B_{N-2}$, quark mixing amplitudes $A$ and flavor $\mathrm{SU}(3)$ breaking parameter $r$. The $N \times N, N \geq 3$ hermitian mass matrix is then diagonalized by the masses of the $N$ physical states:

$$
\begin{gathered}
\left(\begin{array}{cccccccc}
M\left(i_{1}\right) & 0 & \cdot & \cdot & \cdot & 0 & B_{1} & B_{1} r \\
0 & M\left(i_{2}\right) & \cdot & \cdot & \cdot & 0 & B_{2} & B_{2} r \\
\cdot & \cdot & \cdot & & \cdot & \cdot & \cdot \\
\cdot & \cdot & & \cdot & & \cdot & \cdot & \cdot \\
\cdot & \cdot & & & \cdot & \cdot & \cdot & \cdot \\
0 & 0 & \cdot & \cdot & \cdot & M\left(i_{N-2}\right) & B_{N-2} & B_{N-2} r \\
B_{1}^{*} & B_{2}^{*} & \cdot & \cdot & \cdot & B_{N-2}^{*} & M(s \bar{s})+A & A r \\
B_{1}^{*} r^{*} & B_{2}^{*} r^{*} & \cdot & \cdot & \cdot & B_{N-2}^{*} r^{*} & A r^{*} & M(n \bar{n})+A|r|^{2}
\end{array}\right) \\
\Rightarrow \operatorname{diag}\left(\tilde{M}\left(i_{1}\right), \tilde{M}\left(i_{2}\right), \ldots, \tilde{M}\left(i_{N-2}\right), \tilde{M}(\bar{s} s), \tilde{M}(\bar{n} n)\right) .
\end{gathered}
$$

Here we assumed that it is possible to choose a basis where the primitive additional isosinglet states are already diagonalized. For example, if the three isosinglets are a glueball, $c \bar{c}$ and $b \bar{b}$, such a basis can to a good approximation be chosen due to the small mixing between the glueball and hidden flavour heavy quark mesons. We also assumed that the $n \bar{n}$ meson to $s \bar{s}$ meson coupling ratio of all isosinglets is a universal constant $r$. In the limit of $\mathrm{SU}(3)$ symmetry this assumption becomes exact, and $r=\sqrt{2}$.

Since the initial $N \times N$ mass matrix (6) can be rewritten in the following effective $3 \times 3$ form:

$$
\left(\begin{array}{ccc}
\mathbf{M}(i) & \mathbf{B} & \mathbf{B} r \\
\mathbf{B}^{\dagger} & M(s \bar{s})+A & A r \\
\mathbf{B}^{\dagger} r^{*} & A r^{*} & M(n \bar{n})+A|r|^{2}
\end{array}\right)
$$


where

$$
\mathbf{M}(i) \equiv\left(\begin{array}{cccccc}
M\left(i_{1}\right) & 0 & \cdot & \cdot & \cdot & 0 \\
0 & M\left(i_{2}\right) & \cdot & \cdot & \cdot & 0 \\
\cdot & \cdot & \cdot & & \cdot \\
\cdot & \cdot & & \cdot & \cdot \\
\cdot & \cdot & & \cdot & \cdot \\
0 & 0 & \cdot & \cdot & \cdot & M\left(i_{N-2}\right)
\end{array}\right), \quad \mathbf{B} \equiv\left(\begin{array}{c}
B_{1} \\
B_{2} \\
\cdot \\
\cdot \\
\cdot \\
B_{N-2}
\end{array}\right)
$$

it is evident that the general $N \times N$ case effectively reduces to the $3 \times 3$ case considered above. Applying the methods of linear algebra, one can obtain upon some calculation the following most general Schwinger type mass relation for the $N \times N$ case $]^{\text {I }}$

$$
\begin{aligned}
& \prod_{k=1}^{N-2}\left(\left(1+|r|^{2}\right) \tilde{M}\left(i_{k}\right)-|r|^{2} M(\bar{s} s)-M(\bar{n} n)\right)\left(\left(1+|r|^{2}\right) \tilde{M}(\bar{s} s)-|r|^{2} M(\bar{s} s)-M(\bar{n} n)\right) \\
& \times\left(\left(1+|r|^{2}\right) \tilde{M}(\bar{n} n)-|r|^{2} M(\bar{s} s)-M(\bar{n} n)\right) \\
& \quad+\prod_{k=1}^{N-2}\left(\left(1+|r|^{2}\right) M\left(i_{k}\right)-|r|^{2} M(\bar{s} s)-M(\bar{n} n)\right)|r|^{2}(M(\bar{s} s)-M(\bar{n} n))^{2}=0 . \quad(10)
\end{aligned}
$$

In the case of only one additional isosinglet, Eq. (9) reduces to the generalized Schwinger mass formula (4).

\section{The case of an arbitrary number of mesons}

As discussed above, the generalized Schwinger mass formula (10) reduces to the original formula (1) in the limits where the primitive mass of an additional isosinglet goes to infinity, or the isosinglet-meson coupling goes to zero. The main use of the new Schwinger formula is hence where an additional isosinglet is in the vicinity of the mesons, where the conventional Schwinger formula is expected to be inapplicable. However, as the two examples of the $J^{P C}=2^{++}$and $3^{--}$multiplets considered above show, the mass of such an additional isosinglet must generally be expected to be large (in agreement with, e.g., lattice QCD calculations of glueballs [11). Hence the mesons in the new Schwinger formula would need to be radially or orbitally excited mesons, or hybrid mesons (in all nonets except the scalar nonet [12]). It now becomes interesting to consider whether the excited states could influence the mixing of an additional isosinglet with the ground states. It should be decided how many pairs of excited states to incorporate. There are arguments that the effective number of hadronic states of any given type is limited [13]. We shall discuss a 7-state case which is sufficiently complicated to simulate the general case. The $7 \times 7$ matrix has also been discussed in a different context in ref. [14].

Here we investigate a set of three pairs of mesons and test for the effect by choosing an additional isosinglet at various masses. We choose ground states at $M(n \bar{n})=1.2$ $\mathrm{GeV}, M(s \bar{s})=1.5 \mathrm{GeV}$, first excited states at $M^{(1)}(n \bar{n})=1.7 \mathrm{GeV}, M^{(1)}(s \bar{s})=2$

\footnotetext{
${ }^{3}$ One of us (L.B.) wishes to thank C.D. Levermore for a very useful discussion on this point.
} 
$\mathrm{GeV}$, and second excited states at $M^{(2)}(n \bar{n})=2.1 \mathrm{GeV}, M^{(2)}(s \bar{s})=2.4 \mathrm{GeV}$. The mass splittings chosen are typical of constituent quark models [3]. The additional isosinglet mass cases we consider are (i)-(vi). Each of the following two cases,

(i) $M(i)=1.35 \mathrm{GeV}$,

(ii) $M(i)=1.6 \mathrm{GeV}$

simulates the scalar meson case for which an additional isosinglet (the scalar glueball) is in the vicinity of the ground state mesons [12]. Each of the following three cases,

(iii) $M(i)=1.85 \mathrm{GeV}$,

(iv) $M(i)=2.05 \mathrm{GeV}$,

(v) $M(i)=2.25 \mathrm{GeV}$

simulates the tensor meson case for which the mass splitting between the tensor glueball and the $n \bar{n}$ ground state meson is [11] $\sim 0.9 \mathrm{GeV}$. Finally, the case

(vi) $M(i)=2.5 \mathrm{GeV}$

simulates the $2^{-+}$and $3^{--}$cases for which the mass splitting between the corresponding glueball and $n \bar{n}$ ground state meson is [11] $\sim 1.3 \mathrm{GeV}$.

For each of the above six choices of the primitive mass of an additional isosinglet, we first consider the full $7 \times 7$ mass matrix for the mixing of the isosinglet with ground states and both pairs of excited states which generalizes the $3 \times 3$ matrix (5). The couplings of an additional isosinglet to mesons are taken to be the same for ground states and both excited states, for simplicity:(स $B=0.4 \mathrm{GeV}^{2}$, and no direct meson-meson coupling : $A=0$. We then consider separately the mixing of an additional isosinglet with ground states and first and second excited states, each of which is described by the corresponding $3 \times 3$ mass matrix with the same $B=0.4$ $\mathrm{GeV}^{2}$ and $A=0$. In each case, we calculate the masses of all the physical states, using the appropriate quadratic mass relations. Our results in the full $7 \times 7$ case and the separate $3 \times 3$ cases are compared in Table II.

As seen in Table II, the largest discrepancy between the results of the $7 \times 7$ and $3 \times 3$ cases occurs for the $n \bar{n}$ ground state in the case (i) and constitutes $\sim 8 \%$. In every other case, the corresponding discrepancy does not exceed $\sim 2 \%$ (which corresponds to a mass uncertainty of $<50 \mathrm{MeV}$ ), which means that the generalized Schwinger mass formula in every $3 \times 3$ sub-basis should be very accurate. We note that in the actual case of the scalar nonet with smaller $B=0.3 \mathrm{GeV}^{2}$ [12], in the case (i) one would obtain $\tilde{M}(n \bar{n})=1032 \mathrm{MeV}, \tilde{M}(s \bar{s})=1535 \mathrm{MeV}$ for the $7 \times 7$ case, and $\tilde{M}(n \bar{n})=1068 \mathrm{MeV}, \tilde{M}(s \bar{s})=1565 \mathrm{MeV}$ for the $3 \times 3$ case, so that the discrepancy between the results for the $\tilde{M}(n \bar{n})$ reduces to only $\sim 3.5 \%$.

We have also done a similar comparison between the $7 \times 7$ and $3 \times 3$ cases for the same six choices of the primitive mass of an additional isosinglet and the same bare mesons masses, but with nonzero direct meson-meson couplings, $A$, in each of the $2 \times 2$ mesons sub-bases, in addition to nonzero $B$. For $A=B=0.4 \mathrm{GeV}^{2}$, the largest discrepancy between the corresponding results again occurs for the $n \bar{n}$ ground state in the case (i) and constitutes $\sim 7 \%$. In every other case, the corresponding discrepancy does not exceed $\sim 5 \%$ (which corresponds to a mass uncertainty of $<80$

\footnotetext{
${ }^{4}$ This value represents the realistic value of the glueball-meson coupling, as extracted from data by the phenomenological analysis in ref. [15.

${ }^{5}$ It can be shown that there are analytical Schwinger-type formulae in this case [16].
} 
$\mathrm{MeV})$.

Note that in the above analyses, for ground states and both pairs of excited states we formally used a common value of $B$. However, the couplings of the excited states (to the glueball) may be smaller. This would give rise to even smaller errors than quoted above.

On the basis of both possibilities for zero and nonzero $A$ analyzed, we conclude that the generalized Schwinger formula is only weakly affected by the inclusion of the excited states, and must therefore hold with good accuracy for almost every $3 \times 3$ isosinglet-mesons mass sub-matrix.

\section{Conclusions}

Generalized Schwinger mass formulas have been derived for an arbitrary number of isosinglets coupled to a meson nonet. The formulae have significant utility as they mainly refer to experimentally measurable masses, and not unknown couplings; they can therefore be used to predict unknown masses. For real couplings, the generalized Schwinger mass formula with the inclusion of a single isosinglet describes the most general parametrization of a symmetric $3 \times 3$ matrix. In other cases, the matrices are not the most general hermitian matrices, albeit physically relevant ones.

When multiple meson excitations were included in addition to a single isosinglet, we considered the most general matrices. It has been shown for some numerical examples that to a good approximation it is sufficient to restrict to the relevant $3 \times 3$ isosinglet-mesons subspace.

\section{References}

[1] J. Schwinger, Phys. Rev. Lett. 12 (1964) 237

[2] C. Amsler, Rev. Mod. Phys. 70 (1998) 1293

[3] For the most recent review, see S. Godfrey and J. Napolitano, Light meson spectroscopy, hep-ph/9811410, submitted to Rev. Mod. Phys.

[4] For a recent discussion of the use of meson-glueball mass matrices in QCD, see W. Lee and D. Weingarten, IBMHET-98-3, hep-lat/9811024 and references therein.

[5] L. Burakovsky and T. Goldman, hep-ph/9709461; hep-ph/9802404; Phys. Lett. B 427 (1998) 361

[6] Particle Data Group (C. Caso et al.), Eur. Phys. J. C 3 (1998) 1

[7] J. Praschifka, C.D. Roberts and R.T. Cahill, Phys. Rev. D 36 (1987) 209

[8] N.H. Fuchs, Phys. Rev. D 14 (1976) 192

[9] J. Turnau, Z. Phys. C 23 (1984) 89 
[10] H.J. Schnitzer, Nucl. Phys. B 207 (1982) 131

J.L. Rosner, Phys. Rev. D 27 (1983) 1101

[11] S.R. Sharpe, in Proc. of $29^{\text {th }}$ Int. Conf. on High Energy Physics (ICHEP '98), July 1998, Vancouver, Canada, hep-lat/9811006, and references therein

[12] L. Burakovsky and P.R. Page, Phys. Rev. D 59 (1999) 014022, erratum - ibid. 079902.

[13] M.M. Brisudova, L. Burakovsky and T. Goldman, hep-ph/9810296

[14] M. Frank and P.J. O’Donnell, Phys. Rev. D 29 (1984) 921

[15] M.M. Brisudova, L. Burakovsky and T. Goldman, Phys. Rev. D 58 (1998) 114015

[16] L. Burakovsky, P.R. Page, hep-ph/9906282. 


\section{Figure 1}

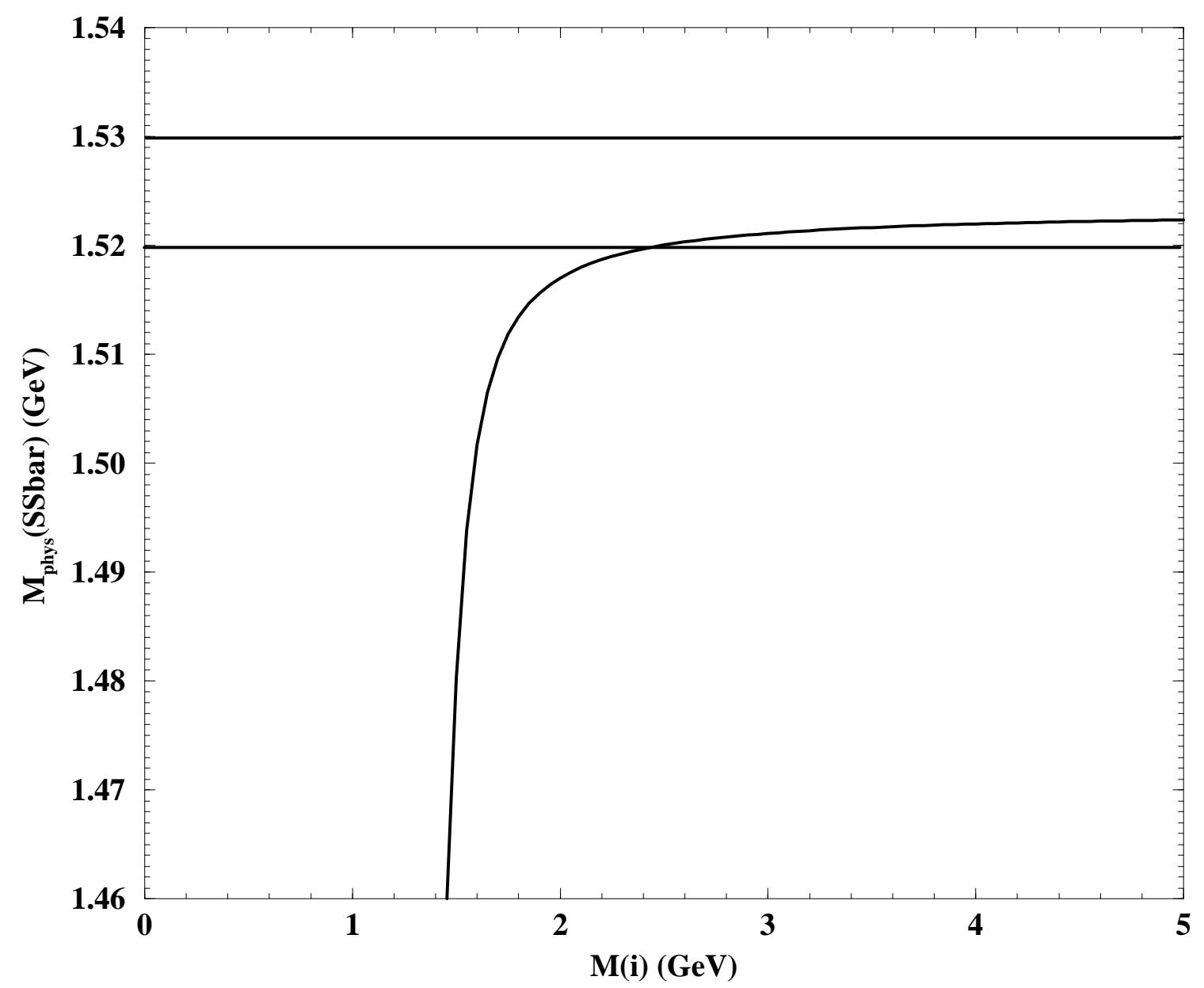

Fig. 1. Comparison of the mass of the physical $s \bar{s}$ state calculated from Eqs. (4),(5) as a function of the bare mass of an additional isosinglet, $M(i)$, with the range allowed by experimental data of ref. [6] (horizontal band), for the $J^{P C}=3^{--}$multiplet. 
Figure 2

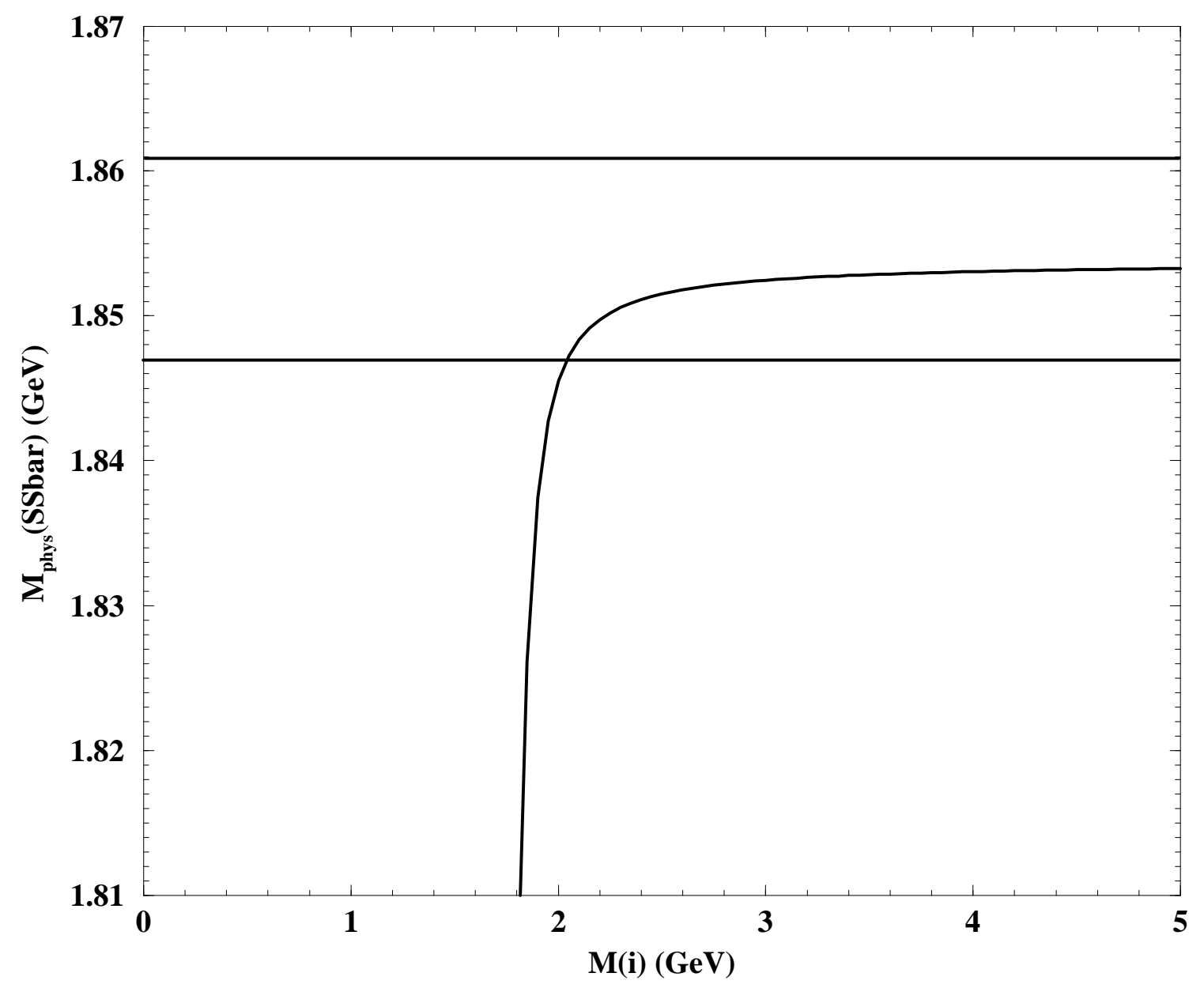

Fig. 2. The same as Fig. 1 for the $J^{P C}=3^{--}$multiplet. 


\begin{tabular}{|c|cc|cc|cc|cc|cc|c|c|}
\hline & \multicolumn{2}{|c|}{ case (i) } & \multicolumn{2}{c|}{ case (ii) } & \multicolumn{2}{c|}{ case (iii) } & \multicolumn{2}{c|}{ case (iv) } & \multicolumn{2}{c|}{ case (v) } & case (vi) \\
\hline & $7 \times 7$ & $3 \times 3$ & $7 \times 7$ & $3 \times 3$ & $7 \times 7$ & $3 \times 3$ & $7 \times 7$ & $3 \times 3$ & $7 \times 7$ & $3 \times 3$ & $7 \times 7$ & $3 \times 3$ \\
\hline$\tilde{M}(n \bar{n})$ & 918 & 994 & 1051 & 1086 & 1115 & 1131 & 1141 & 1150 & 1157 & 1162 & 1169 & 1171 \\
$\tilde{M}(s \bar{s})$ & 1545 & 1607 & 1405 & 1444 & 1452 & 1468 & 1470 & 1478 & 1479 & 1484 & 1486 & 1488 \\
\hline$\tilde{M}^{(1)}(n \bar{n})$ & 1765 & 1763 & 1802 & 1802 & 1617 & 1581 & 1645 & 1632 & 1662 & 1657 & 1674 & 1671 \\
$\tilde{M}^{(1)}(s \bar{s})$ & 2015 & 2020 & 2019 & 2030 & 2028 & 2065 & 1929 & 1947 & 1964 & 1973 & 1981 & 1985 \\
\hline$\tilde{M}^{(2)}(n \bar{n})$ & 2134 & 2127 & 2148 & 2135 & 2179 & 2155 & 2235 & 2195 & 2058 & 2015 & 2073 & 2060 \\
$\tilde{M}^{(2)}(-\bar{s} \bar{s})$ & 2410 & 2409 & 2412 & 2411 & 2418 & 2415 & 2428 & 2423 & 2463 & 2446 & 2373 & 2364 \\
\hline$\tilde{M}(i)$ & 1344 & $1342 \pm 51$ & 1574 & $1631 \pm 96$ & 1870 & $1841 \pm 76$ & 2042 & $2007 \pm 87$ & 2317 & $2292 \pm 14$ & 2603 & $2544 \pm 23$ \\
\hline
\end{tabular}

